\title{
Dynamism of Antimicrobial Activity of Actinomycetes-A Case Study from Undisturbed Microbial Niche
}

\author{
Jayshree D. Patel1, Mahesh Parmar'2, Purshottam Patel ${ }^{2}$, Pravin Rohit ${ }^{2}$, Ramesh Taviyad ${ }^{2}$, \\ Parveen Ansari², Bipasha Bhattacharya², Dhaval Vyas², Vipin Kumar'1, Nirmal S. Sahay², \\ Pawan K. Singh ${ }^{*}$ \\ ${ }^{1}$ National Innovation Foundation India, Satellite Complex, Nr. Mansi Circle, Ahmedabad, Gujarat, India \\ ${ }^{2}$ Sadbhav SRISTI Sanshodhan Laboratory, AES Boys Hostel Campus, Nr. Gujarat University Library \& SBI Bank, \\ Navrangpura, Ahmedabad, Gujarat, India \\ Email: "pawan@nifindia.org
}

Received 15 February 2014; revised 15 March 2014; accepted 30 March 2014

Copyright (C) 2014 by authors and Scientific Research Publishing Inc.

This work is licensed under the Creative Commons Attribution International License (CC BY). http://creativecommons.org/licenses/by/4.0/

c) (i) Open Access

\begin{abstract}
Escalation of drug resistant microbes (bacteria) had forced researchers to search new and improved therapeutic compounds from different possible sources, including metabolites secreted by the actinomycetes. The aim of this study was to evaluate the pattern of antimicrobial actinomycetes from physiologically distinct soil of different geographical locations. Forty five soil samples were collected from 5 districts of Gujarat including two sanctuaries as source of survey for bioactive actinomycetes. Crowded plate technique was used for isolation and Agar cylinder method was employed for the antimicrobial screening. A total of 171 actinomycetes were isolated and screened against eighteen pathogens responsible for causing diseases in plants and humans. Results indicate that $79 \%$ of the isolates were active against at least one of the eighteen tested pathogens. Some of the actinomycetes strain had shown strong antibacterial and antifungal activity which may be a good source of obtaining novel antimicrobials.
\end{abstract}

\section{Keywords}

Actinomycetes, Screening, Antimicrobial, Drug Resistance, Soil Microflora

\section{Introduction}

Actinomycetes, a gram positive filamentous bacterium, are well known for their unique ability to produce vari-

"Corresponding author. 
ous bioactive compounds used as antibiotics [1]-[4]. In recent years discovery of bioactive compounds from actinomycetes is getting momentum due to the ever increasing multi and extensively drug resistant pathogens. Unexplored habitat and niche attracts attention for the isolation and screening of bioactive novel actinomycetes. A study on dynamics of bioactive actinomycetes in different untouched areas of Gujarat India was conducted with an objective to examine the populations in conserved habitats and to evaluate their antimicrobial properties. Antimicrobial activity and population dynamics of the actinomycetes isolated from studied area are not yet reported in any published literature.

Historically, most commonly soil isolated actinomycetes have been of genus Streptomycetes and Micromonospora [5]. Compounds isolated from actinomycetes have numerous chemical structures. The macrolides, tetracyclines, aminoglycosides, glycopeptides and ansamicines are used in antibacterial treatments whereas anthracyclines reached the market to supplement anticancer chemotherapy. The fairly toxic polyether-type antibiotics are used as anti-coccicidal agents. Today about 130 to 140 microbial products are applied in human and veterinary medications and about 15 to 20 compounds are used in agriculture mainly as pesticides, plant protecting agents and food additives [6]. Out of all the novel bioactive metabolites, about 70\% were derived from actinomycetes [7]. Due to intensely increasing resistance of clinically important bacterial strains, discovery of novel types of antibacterial agents is urgently needed. Microbial screening continues to represent an important route for discovery of novel chemicals for the development of therapeutic agents [8]. Thus, it is necessary to continue screening for new metabolites and evaluate the potential of less known and new microbial taxa for development of novel and improved compounds for future use especially against drug-resistant bacteria [9]. Screening of the actinomycetes for the production of novel antibiotics has been intensively pursued by many scientists [10] [11]. Purpose of this study was to isolate new actinomycetes from untouched area of Gujarat and evaluate their antimicrobial potential to develop new bioactive formulations for control of microbial pathogens.

\section{Material and Methods}

\subsection{Soil Samples}

Samples were collected from 5 districts of Gujarat, from various locations viz., grassland, hills, river side, nursery etc. The samples were collected separately from the depth of 10 - $15 \mathrm{~cm}$ in a clean, dry and sterile polythene bags with the help of sterile open-end soil borer. Characteristically distinct sites on the basis of soil organic matter and moisture content, particle size and colour were selected for sampling. Appropriate sterile materials were used and sterile conditions were maintained during the study.

\subsection{Test Cultures}

Eighteen pathogenic microbial cultures (10 bacteria and 8 fungi) were procured from MTCC, Chandigarh. Bacterial pathogens were Staphylococcus aureus (MTCC 96), Pseudomonas aeruginosa (MTCC 741), Escherichia coli (MTCC 443), Xanthomonas campestris (MTCC 2286), Erwinia carotovora (MTCC 1428), Listeria monocytogenes (MTCC 657), Bacillus subtilis (MTCC 441), Yersinia enterocolitica (MTCC 861), Salmonella typhimurium(MTCC 98) and Vibrio vulnificus (MTCC 1145); 8 fungal pathogens were Candida albicans (MTCC 183), Fusarium oxysporum (MTCC 284), Rhizoctonia oryzae-sativae (MTCC 2162), Trichophyton rubrum (MTCC 296), Acremonium recifei (MTCC 3570), Microsporum gypseum (MTCC 2830), Malassezia furfur (MTCC 1374) and Ustilago maydis (MTCC 1474). All the microbial isolates were sub-cultured on synthetic medium according to their specification. The bacterial pathogens and C. albicans were sub-cultured on Nutrient agar media (Hi-Media MM 002) 18 hrs before and incubated at $30^{\circ} \mathrm{C}$ in order to get actively growing culture. The fungal cultures were inoculated on Sabouraud dextrose agar (SDA, Hi-Media M 033) plate 3 days earlier and incubated at room temperature for activation.

\subsection{Isolation of Actinomycetes}

Crowded plate technique [12] was employed for the isolation of actinomycetes from collected soil samples. Samples were air dried at room temperature for 10 - 14 days, passed through a $0.8 \mathrm{~mm}$ mesh and preserved in sterile polythene bags for further use. For serial dilution, $1 \mathrm{~g}$ each of the soil samples were diluted 10 times in sterile physiological water $\left(\mathrm{NaCl} 9 \mathrm{gL}^{-1}\right)$ and homogenized by vortexing for $10-15 \mathrm{~min}$ [13]. Portion of $1 \mathrm{ml}$ soil suspensions were transferred to $9 \mathrm{ml}$ sterile distilled water and subsequently diluted to $10^{2}, 10^{3}, 10^{4}, 10^{5}$, and 
$10^{6}$. Isolation was carried out on Actinomycetes Isolation Agar (AIA, HiMedia MM 012). Petri plates were incubated at $28^{\circ} \mathrm{C}$ for 14 - 21 days for proper growth. Seventh day onwards, isolated actinomycetes colonies were re-plated on AIA medium and incubated at $28^{\circ} \mathrm{C}$. Pure actinomycetes isolates were maintained on AIA at $4^{\circ} \mathrm{C}$ in refrigerator for further experimentation. To determine antimicrobial activities, Actinomycetes were revived by streaking on AIA and incubated at $28^{\circ} \mathrm{C}$ for 7 days.

\subsection{Antimicrobial Activity}

Antimicrobial activity was estimated by Agar cylinder method [14]. From well grown culture, five discs (6 mm in diameters) were cut and placed on freshly inoculated bacterial pathogen (0.5 McFarland) on Muller-Hinton agar (MHA, HiMedia M 173) and fungal pathogens were inoculated on Sabouraud dextrose Agar (SDA, HiMedia M 033) supplemented with 3\% agar using spore suspension. Plates were first kept at low temperature for at least 2 hours to allow the diffusion of produced antibiotics. For bacteria and yeast cultures the plates were incubated at $37^{\circ} \mathrm{C}$ and inhibition was recorded after 24 hours. For fungi the plates were incubated at $28^{\circ} \mathrm{C}$ and inhibition was recorded after 72 hours. The activities were recorded by measuring the diameter of zone of inhibition in nearest mm using antibiotic zone reader.

\section{Results and Discussion}

\subsection{Isolation of Actinomycetes}

Many researchers have isolated actinomycetes from various natural sources like soil [15]-[17], mud [18], marine [19]-[21], plants [22] [23], termite guts [24] which are capable to produce novel bioactive compounds and are also compatible antimicrobial agents for healing of the infections [25] [26]. In the present study, Actinomycetes were isolated from 45 soil samples collected from 5 different regions of Gujarat mainly from the untouched area. Sampling was performed from different soil types like sandy, sandy loam, loamy, loamy clay and clay with either little or no organic matter. Total 171 morphologically distinct actinomycetes were isolated and preserved for antimicrobial screening. Most of the strains were isolated on AIA selective medium. This medium seems to be most specific and sensitive for actinomycetes isolation as it contains sodium propionate which acts as antifungal agent [13]. All of the actinomycetes were isolated at mesophilic temperature $\left(28^{\circ} \mathrm{C}\right)$. The result indicates a dynamic population diversity of actinomycetes (Table 1). Some of the species were isolated from more than 10 different locations. Homogeneous, heterogeneous and unique population pattern was observed. Out of total 171 isolates, 89 isolates were found uniquely distributed in single location whereas 82 isolates were found dominant in more than two locations. Maximum of 39 actinomycetes were isolated from the site 36 followed by 27 from site 39 and 26 from site 40 (Figure 1). Minimum type (1 type) of actinomycetes was isolated from site 3, 4 and 10 respectively. Soil with different texture contains variable characteristics of actinomycetes isolates [27]-[29]. Two isolates were found present at 12 out of 45 locations, though they had not shown promising antimicrobial activity as compared to other isolates; whereas one isolate had shown antimicrobial activity against all the 18 screened pathogens which was found at only one location.

\subsection{Antibiotic Production}

The results of antimicrobial screening of 171 actinomycetes showed that 134 (79\%) had inhibitory activity against one or more pathogens. Figure 2 illustrates that out of 171 actinomycetes isolated, $36(21.05 \%)$ had not shown any antimicrobial activity against 17 selected pathogens whereas $1(0.58 \%)$ was having antimicrobial activity against all the 17 pathogens. Five isolates (2.92\%) had shown activity against 14 pathogens, 4 (2.33\%) against 12 pathogens, 5 (2.92\%) against 11 pathogens, 7 (4.09\%) against 10 pathogens, 6 (3.50\%) against 9 pathogens, 5 (2.92\%) against 8 pathogens, 7 (4.09\%) against 7 pathogens, 7 (4.09\%) against 6 pathogens, 15 (8.77\%) against 5 pathogens, 5 (2.92\%) against 4 pathogens, 13 (7.06\%) against 3 pathogens, 25 (14.61\%) against 2 pathogens and 30 (17.54\%) against 1 pathogen. Analysis of the results indicates that 90 (53\%) Actinomycetes do not have any activity against the fungal pathogens whereas in case of bacterial pathogens this number was 49 (29\%). Percentage of actinomycetes strains producing antimicrobial compounds in standard agar diffusion assay ranges between $30 \%$ to $80 \%$ depending on the ecological and taxonomical groups [30]. Search for the novel metabolites especially from actinomycetes requires screening from large number of isolates in order to discover novel compounds of pharmaceutical important. Adaptation in term led the actinomycetes to produce their own secondary metabolites to survive [15]. 
Table 1. The table represents the frequency of actinomycetes their \%, total number of sites from where they were isolated, their number and \% of sampling sites along with remarks.

\begin{tabular}{|c|c|c|c|c|c|c|c|}
\hline $\begin{array}{l}\text { Frequency } \\
\text { of } \\
\text { Occurrence }\end{array}$ & Actinomycetes & $\begin{array}{l}\text { No. of } \\
\text { Actinomycetes }\end{array}$ & $\begin{array}{c}\% \text { of } \\
\text { Actinomycetes }\end{array}$ & No. of Sites & $\begin{array}{l}\text { Total } \\
\text { No. of } \\
\text { Sites }\end{array}$ & $\begin{array}{l}\% \text { of } \\
\text { Sites }\end{array}$ & Remarks \\
\hline 1 & $\begin{array}{l}\text { SAct001, SAct002, SAct004, } \\
\text { SAct005, SAct006, SAct007, } \\
\text { SAct008, SAct014, SAct017, } \\
\text { SAct018, SAct019, SAct021, } \\
\text { SAct026, SAct030, SAct034, } \\
\text { SAct035, SAct037, SAct040, } \\
\text { SAct041, SAct043, SAct046, } \\
\text { SAct050, SAct051, SAct053, } \\
\text { SAct054, SAct055, SAct059, } \\
\text { SAct063, SAct065, SAct066, } \\
\text { SAct069, SAct073, SAct074, } \\
\text { SAct075, SAct076, SAct096, } \\
\text { SAct102, SAct105, SAct106, } \\
\text { SAct111, SAct112, SAct113, } \\
\text { SAct115, SAct117, SAct120, } \\
\text { SAct121, SAct122, SAct123, } \\
\text { SAct125, SAct126, SAct128, } \\
\text { SAct129, SAct130, SAct131, } \\
\text { SAct132, SAct133, SAct134, } \\
\text { SAct135, SAct136, SAct137, } \\
\text { SAct138, SAct141, SAct142, } \\
\text { SAct143, SAct145, SAct146, } \\
\text { SAct148, SAct149, SAct150, } \\
\text { SAct151, SAct152, SAct153, } \\
\text { SAct154, SAct155, SAct156, } \\
\text { SAct157, SAct158, SAct160, } \\
\text { SAct161, SAct162, SAct163, } \\
\text { SAct164, SAct165, SAct166, } \\
\text { SAct167, SAct168, SAct169, }\end{array}$ & 89 & 52.65 & $\begin{array}{c}\text { 8,13,14,21, } \\
22,24,25,27,2 \\
9,31,32,33,34, \\
36,37,38,39,4 \\
0,42,43,44,45\end{array}$ & 22 & 48.89 & $\begin{array}{l}\text { These actinomycetes } \\
\text { were unique in } \\
\text { morphological } \\
\text { characters and there was } \\
\text { no repeation of this at } \\
\text { other sites. They were } \\
\text { found at only one } \\
\text { location. This represents } \\
\text { 48.89\% of the samples. }\end{array}$ \\
\hline 2 & $\begin{array}{l}\text { SAct011, SAct012, SAct023, } \\
\text { SAct024, SAct025, SAct027, } \\
\text { SAct036, SAct038, SAct045, } \\
\text { SAct047, SAct056, SAct060, } \\
\text { SAct061, SAct064, SAct068, } \\
\text { SAct071, SAct077, SAct081, } \\
\text { SAct087, SAct094, SAct097, } \\
\text { SAct099, SAct107, SAct108, } \\
\text { SAct109, SAct110, SAct127, } \\
\text { SAct147 }\end{array}$ & 28 & 16.56 & $\begin{array}{c}13,14,16,17,2 \\
2,23,24,25,26, \\
27,28,29,30,3 \\
1,32,34,35,36, \\
37,38,39,40,4 \\
1,42\end{array}$ & 24 & 53.33 & $\begin{array}{l}\text { These actinomycetes } \\
\text { were found at two } \\
\text { locations. This } \\
\text { represents } 53.33 \% \text { of } \\
\text { the samples. }\end{array}$ \\
\hline 3 & $\begin{array}{l}\text { SAct009, SAct015, SAct022, } \\
\text { SAct028, SAct032, SAct042, } \\
\text { SAct044, SAct048, SAct049, } \\
\text { SAct052, SAct062, SAct070, } \\
\text { SAct072, SAct078, SAct093, } \\
\text { SAct098, SAct101, SAct103, } \\
\text { SAct104, SAct118, SAct124, } \\
\text { SAct140, SAct159 }\end{array}$ & 23 & 13.6 & $\begin{array}{c}1,7,10,12,13,1 \\
4,15,16,18,20, \\
21,22,23,24,2 \\
5,26,27,28,29, \\
31,32,33,34,3 \\
6,38,39,40,41, \\
42\end{array}$ & 29 & 64.44 & $\begin{array}{l}\text { These actinomycetes } \\
\text { were observed at three } \\
\text { locations. This } \\
\text { represents } 64.44 \% \text { of } \\
\text { the samples. }\end{array}$ \\
\hline 4 & $\begin{array}{l}\text { SAct003, SAct020, SAct029, } \\
\text { SAct033, SAct058, SAct067, } \\
\text { SAct086, SAct100, SAct119 }\end{array}$ & 9 & 5.32 & $\begin{array}{l}2,7,10,13,14,1 \\
8,20,21,22,23, \\
24,25,32,34,3 \\
6,38,39,40,42\end{array}$ & 19 & 42.22 & $\begin{array}{l}\text { These actinomycetes } \\
\text { were found at } 4 \\
\text { locations. This } \\
\text { represents } 42.22 \% \text { of } \\
\text { the samples. }\end{array}$ \\
\hline 5 & $\begin{array}{l}\text { SAct016, SAct031, SAct057, } \\
\text { SAct079, SAct080, SAct084, } \\
\text { SAct114, SAct116 }\end{array}$ & 8 & 4.73 & $\begin{array}{c}2,5,6,7,10,11, \\
13,14,15,17,1 \\
8,19,21,25,29, \\
32,34,36,38,3 \\
9,40,41\end{array}$ & 22 & 48.89 & $\begin{array}{l}\text { These actinomycetes } \\
\text { were found at } 5 \\
\text { locations. This } \\
\text { represents } 48.89 \% \text { of } \\
\text { the samples. }\end{array}$ \\
\hline 6 & $\begin{array}{c}\text { SAct090, SAct092, SAct095, } \\
\text { SAct139 }\end{array}$ & 4 & 2.36 & $\begin{array}{c}1,5,7,13,14, \\
15,16,18,19,2 \\
1,22,25,29,36, \\
38,39,40,42\end{array}$ & 18 & 40.00 & $\begin{array}{l}\text { These actinomycetes } \\
\text { were observed at } 6 \\
\text { locations. This } \\
\text { represents } 40.00 \% \text { of } \\
\text { the samples. }\end{array}$ \\
\hline
\end{tabular}




\begin{tabular}{|c|c|c|c|c|c|c|c|}
\hline \multicolumn{8}{|c|}{ Continued } \\
\hline 7 & SAct010, SAct082, SAct091 & 3 & 1.77 & $\begin{array}{c}1,5,6,7,10 \\
11,15,18,20 \\
22,24,25,29 \\
32,36,38,39\end{array}$ & 17 & 37.78 & $\begin{array}{l}\text { These actinomycetes } \\
\text { were isolated from } 7 \\
\text { locations. This } \\
\text { represents } 37.78 \% \text { of } \\
\text { the samples. }\end{array}$ \\
\hline 9 & SAct039, SAct088 & 2 & 1.18 & $\begin{array}{c}1,6,8,9,12,13 \\
14,15,16,18 \\
21,22,23,25 \\
38\end{array}$ & 15 & 33.33 & $\begin{array}{l}\text { These actinomycetes } \\
\text { were isolated from } 9 \\
\text { locations. This } \\
\text { represents } 33.33 \% \text { of } \\
\text { the samples. }\end{array}$ \\
\hline 10 & SAct085 & 1 & 0.59 & $\begin{array}{c}6,7,10,12,13 \\
18,21,25,38 \\
41\end{array}$ & 10 & 22.22 & $\begin{array}{l}\text { These actinomycetes } \\
\text { were isolated from } \\
10 \text { locations. This } \\
\text { represents } 22.22 \% \text { of } \\
\text { the samples. }\end{array}$ \\
\hline 11 & SAct083, SAct144 & 2 & 1.18 & $\begin{array}{l}4,5,6,7,8,10 \\
11,13,15,18, \\
21,23,25,26 \\
36,38,39,42\end{array}$ & 18 & 40.00 & $\begin{array}{l}\text { These actinomycetes } \\
\text { were isolated from } \\
11 \text { locations. This } \\
\text { represents } 40.00 \% \text { of } \\
\text { the samples. }\end{array}$ \\
\hline 12 & SAct013, SAct089 & 2 & 1.18 & $\begin{array}{c}6,7,10,12,13 \\
14,16,17,18 \\
19,20,21,23, \\
25,29,34,36 \\
38,40\end{array}$ & 19 & 42.22 & $\begin{array}{l}\text { These actinomycetes } \\
\text { were isolated from } \\
12 \text { locations. This } \\
\text { represents } 42.22 \% \text { of } \\
\text { the samples. }\end{array}$ \\
\hline
\end{tabular}

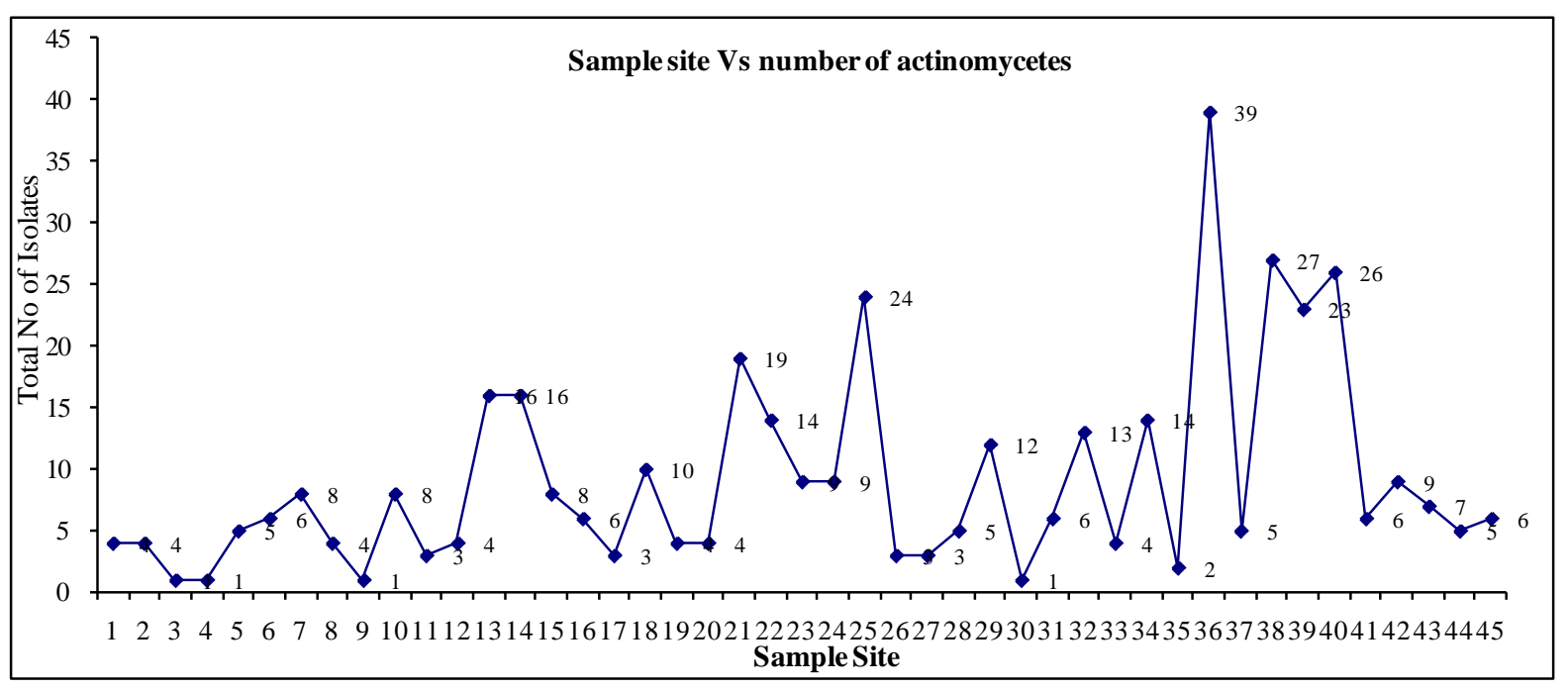

Figure 1. The number of actinomycetes isolated from each soil samples is represented in the graph. Y-axis depicts the number of actinomycetes and $\mathrm{X}$-axis represents sample number.

From the experiments it was found that, 52 actinomycetes were able to control the growth of plant pathogenic bacteria Erwinia carotovora, 58 were found inhibitory to Bacillus subtilis, 51 had shown inhibition against Escherichia coli, 59 against Staphylococcus aureus, 54 against Xanthomonas campestris, 48 against Vibrio vulnificus, 29 against Salmonella typhimurium, 44 against Listeria monocytogenes, 76 against Yersinia enterocolitica, 23 against highly resistant strain of Pseudomonas aeruginosa; 13 against fungi Acremonium recifei; 30 against Ustilago maydis, 25 against Microsporum gypseum, 13 against Candida albicans, 21 against Rhizoctonia oryzae-sativae, 21 against Fusarium oxysporum, 34 against Trichophyton rubrum and 7 against Malassezia furfur (Table 2). Roughly 65\% of the graph area in Figure 3 is showing antibacterial, $15 \%$ antifungal and $20 \%$ without any activity respectively. This clearly demonstrates the richness of the sites for the microbes with antimicrobial property. 
Table 2. Shows the antimicrobial activity of Actinomycetes against different pathogens with the intensity of the zone of inhibition including the disc diameter. Very strong: >46 mm; Strong: 26 - 46 mm; Medium: 10 - 25 mm; Low: $<10$ mm.

\begin{tabular}{|c|c|c|c|c|c|c|c|}
\hline \multirow{2}{*}{$\begin{array}{l}\text { Sr. } \\
\text { No. }\end{array}$} & \multirow{2}{*}{ Name of pathogen } & \multirow{2}{*}{ Causative agent for } & \multicolumn{5}{|c|}{ Types of effectiveness of Actinomycetes } \\
\hline & & & Total & Very strong & Strong & Medium & Low \\
\hline 1 & Erwinia carotovora & $\begin{array}{l}\text { Bacterial soft rot in } \\
\text { carrots }\end{array}$ & 52 & 8 & 16 & 23 & 5 \\
\hline 2 & Bacillus subtilis & Dysentery & 58 & 1 & 12 & 38 & 7 \\
\hline 3 & Escherichia coli & $\begin{array}{l}\text { Extra intestinal } \\
\text { infections }\end{array}$ & 51 & 1 & 9 & 28 & 13 \\
\hline 4 & Staphylococcus aureus & $\begin{array}{l}\text { Acute food poisons \& } \\
\text { skin infections }\end{array}$ & 59 & 2 & 8 & 33 & 16 \\
\hline 5 & Xanthomonas campestris & $\begin{array}{c}\text { Black rot in cruciferous } \\
\text { plants }\end{array}$ & 54 & 1 & 11 & 24 & 18 \\
\hline 6 & Vibrio vulnificus & $\begin{array}{l}\text { Fatal septicaemia, } \\
\text { Gastroenteritis }\end{array}$ & 48 & 3 & 11 & 29 & 5 \\
\hline 7 & Salmonella typhimurium & $\begin{array}{c}\text { Typhoid, Gastrointestinal } \\
\text { problem }\end{array}$ & 29 & 0 & 7 & 15 & 7 \\
\hline 8 & Listeria monocytogenes & $\begin{array}{c}\text { Listeriosis, Poultry } \\
\text { diseases }\end{array}$ & 44 & 2 & 15 & 21 & 6 \\
\hline 9 & Yersinia enterocolitica & $\begin{array}{l}\text { Septicaemia, Acute } \\
\text { appendicitis }\end{array}$ & 76 & 0 & 34 & 29 & 13 \\
\hline 10 & Pseudomonas aeruginosa & Post surgery infections & 23 & 0 & 1 & 10 & 12 \\
\hline 11 & Acremonium recifei & White grain Mycetoma & 13 & 0 & 0 & 9 & 4 \\
\hline 12 & Ustilago maydis & Corn smut & 30 & 0 & 4 & 16 & 10 \\
\hline 13 & Malassezia furfur & $\begin{array}{l}\text { Superficial fungal } \\
\text { disease of skin }\end{array}$ & 7 & 0 & 0 & 0 & 7 \\
\hline 14 & Microsporum gypseum & Degradation of Keratin. & 25 & 0 & 2 & 9 & 14 \\
\hline 15 & Candida albicans & $\begin{array}{l}\text { Skin and pediatric } \\
\text { infection }\end{array}$ & 13 & 0 & 0 & 6 & 7 \\
\hline 16 & Fusarium oxysporum & Fusarium wilt & 21 & 0 & 0 & 13 & 8 \\
\hline 17 & Rhizoctonia oryzae-sativa & Root rot and sheath spot & 21 & 0 & 6 & 7 & 8 \\
\hline 18 & Trichophyton rubrum & Dermetomycoses & 34 & 2 & 3 & 16 & 13 \\
\hline
\end{tabular}

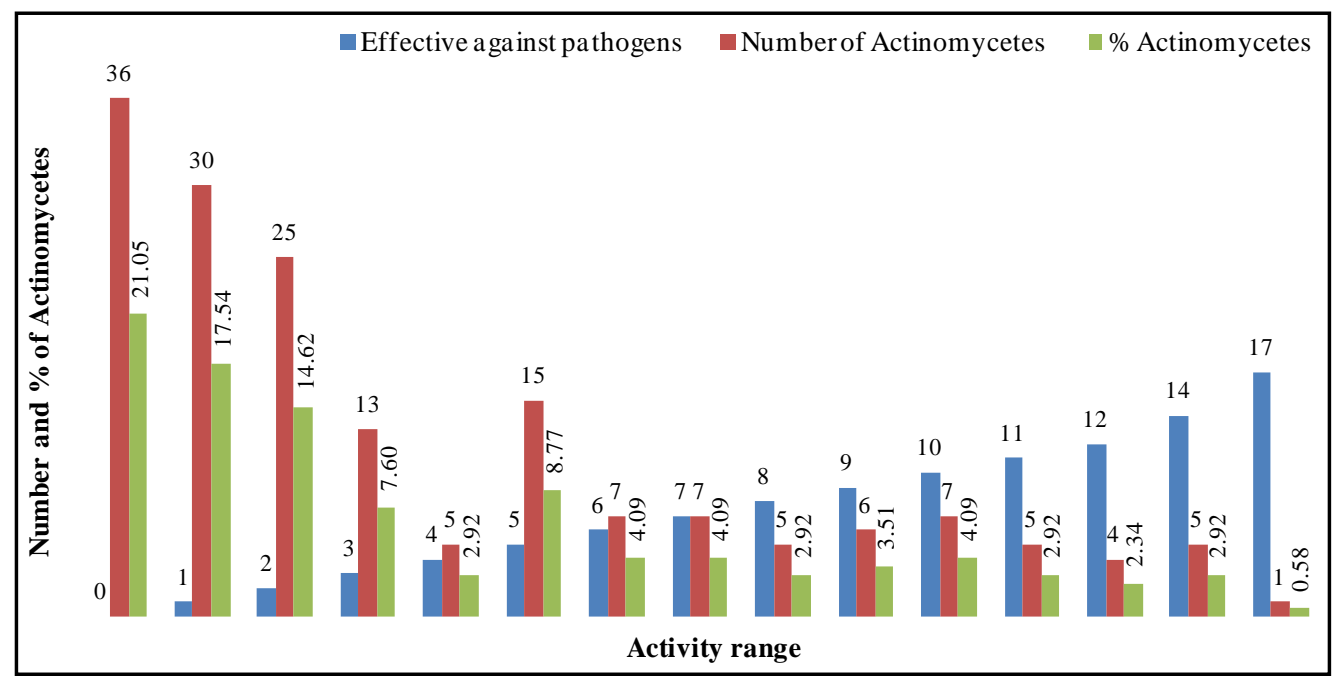

Figure 2. Represents the Number and per cent of Actinomycetes showing activity on Y-axis where as $\mathrm{X}$-axis shows the respective number of pathogens as $1=0,2=1,3=2,4=3,5=4,6=5,7=6,8=7$, $9=8,10=9,11=10,12=11,13=12,14=14$ and $15=17$ respectively. 


\section{Conclusion}

One hundred and seventy one actinomycetes strains were isolated from different untouched regions of Gujarat. Screening was performed against 18 plant and human pathogenic bacteria and fungi. Isolates producing bioactive compounds were expressed by their antibiotic activity spectrum. Extensive screening of the isolates for their antibacterial and antifungal activity revealed that they have strong antibiotic producing potential. The study implies that actinomycetes from Gujarat soil are a potential source for development of novel anti-microbial agents.

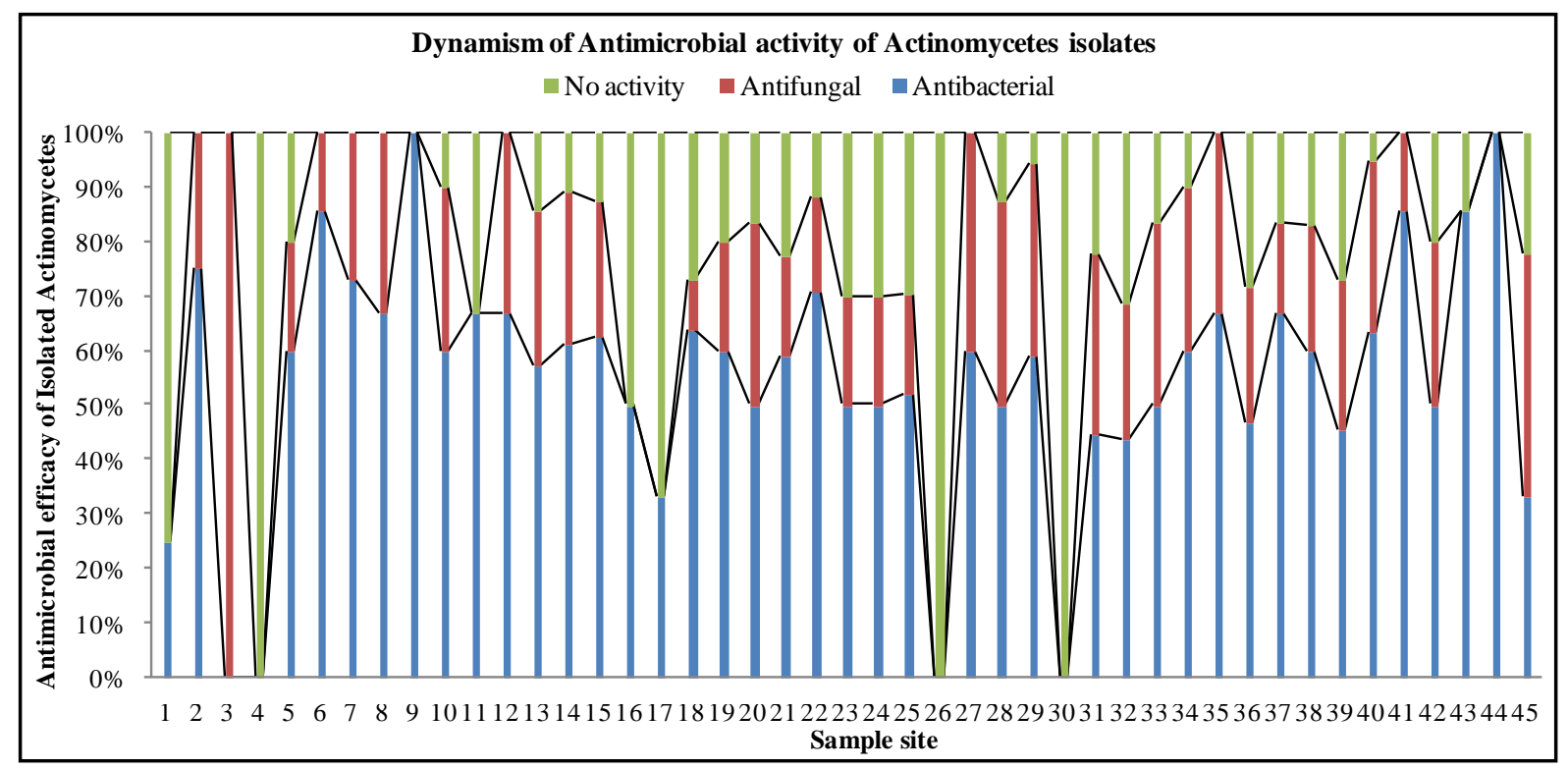

Figure 3. Represents the actinomycetes of each sampling sites along with their antibacterial, antifungal and no activity against pathogenic bacteria and fungi tested. Roughly 65 percent of the graph area is showing antibacterial, 15 percent antifungal and 20 percent without any activity respectively. The actinomycetes of 3 sampling sites (4, 26 and 30) had not shown any antimicrobial activity whereas 2 sampling sites ( 9 and 44$)$ had shown 100 percent antibacterial activity.

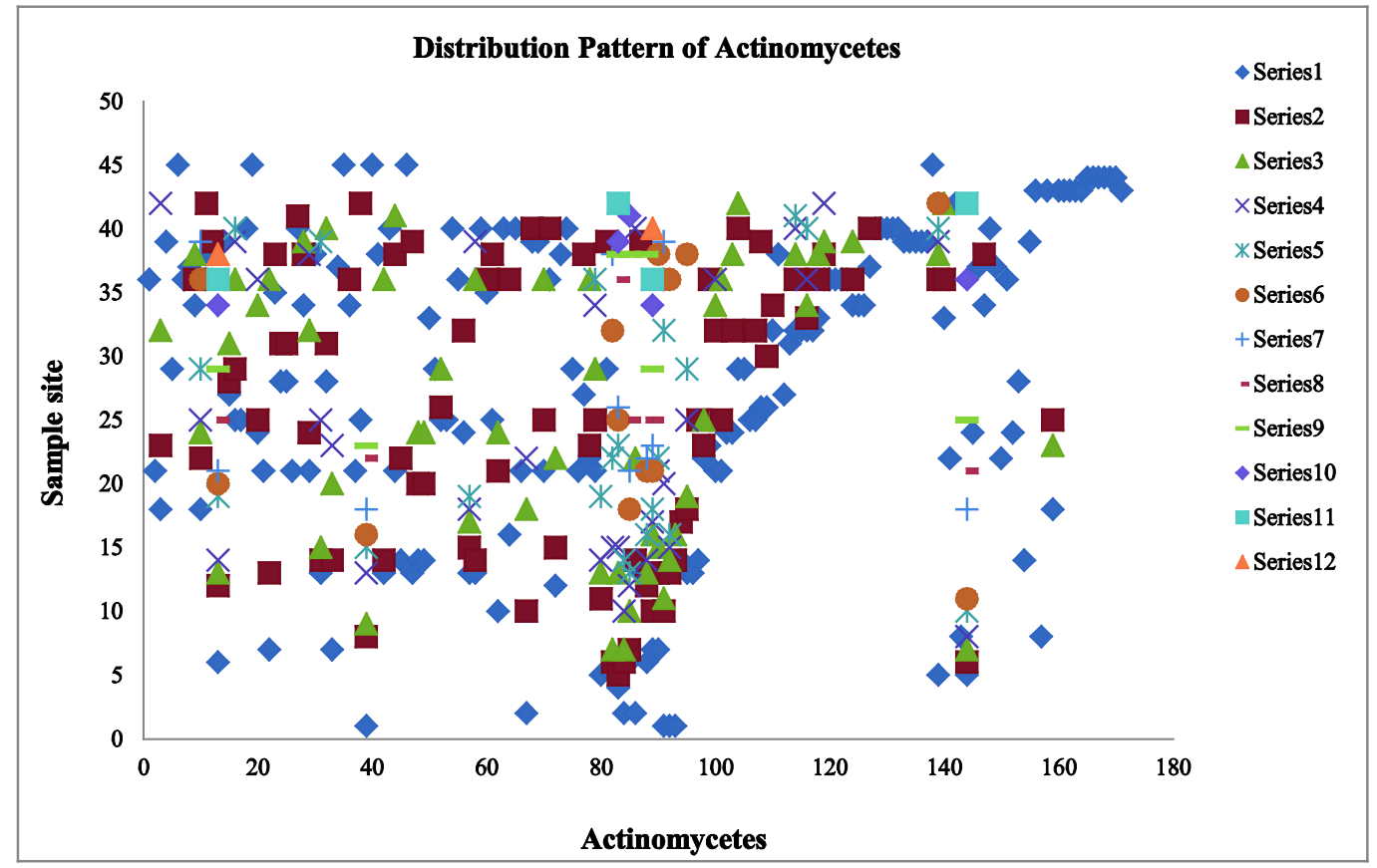

(a) 


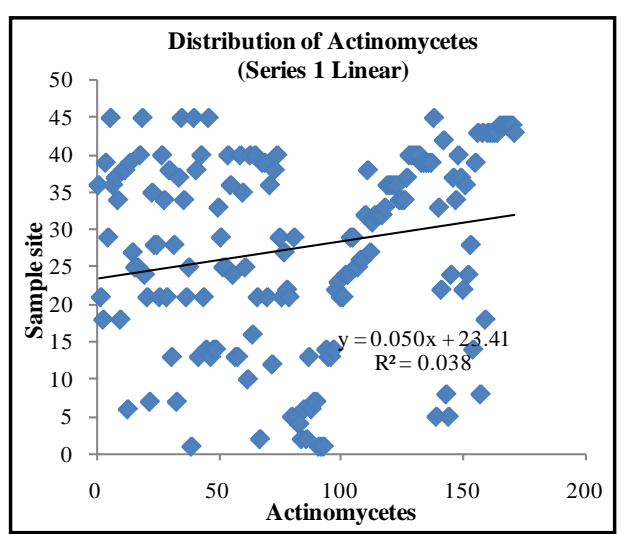

(b)

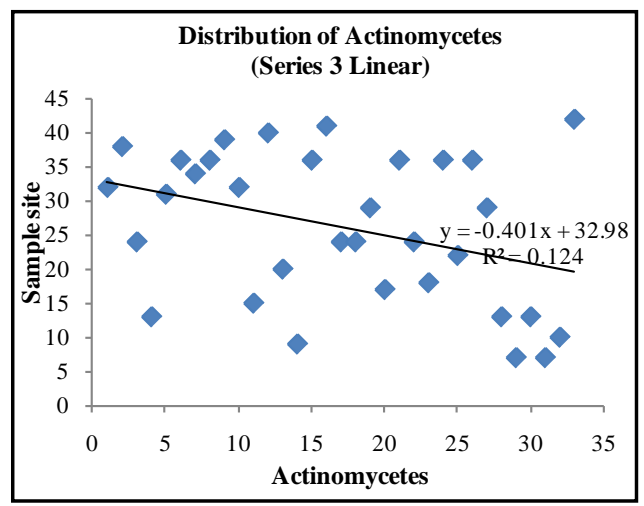

(d)

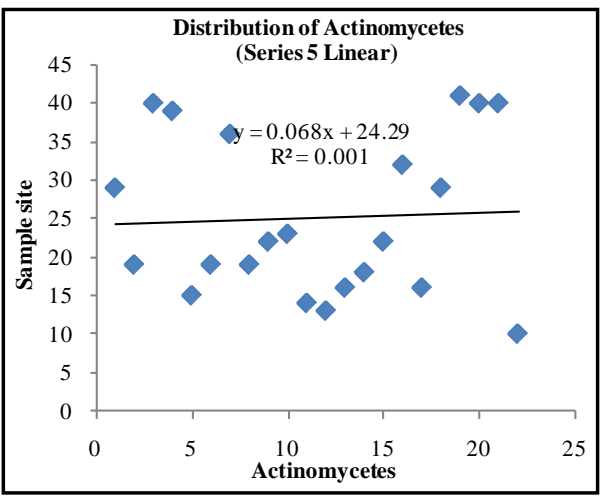

(f)

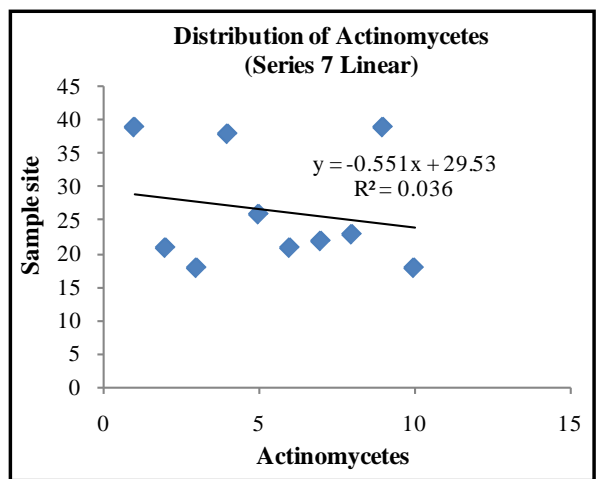

(h)

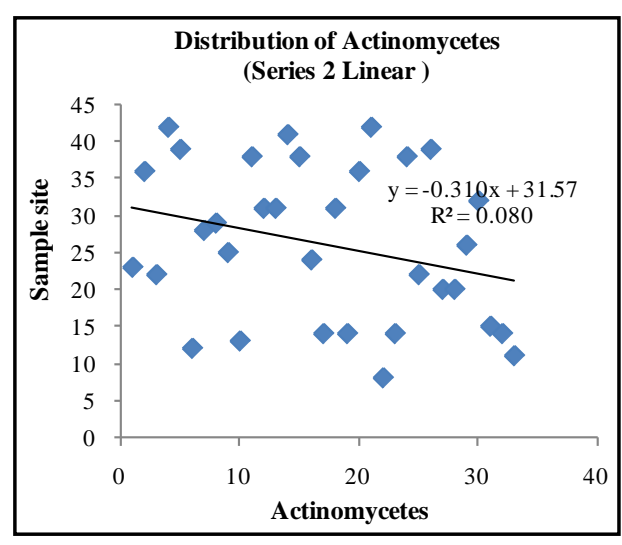

(c)

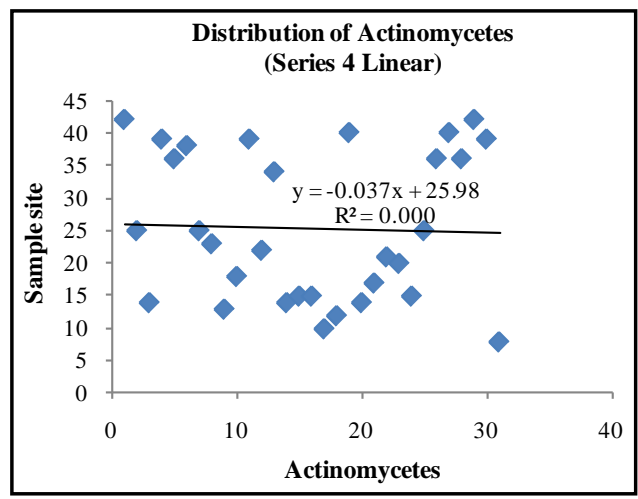

(e)

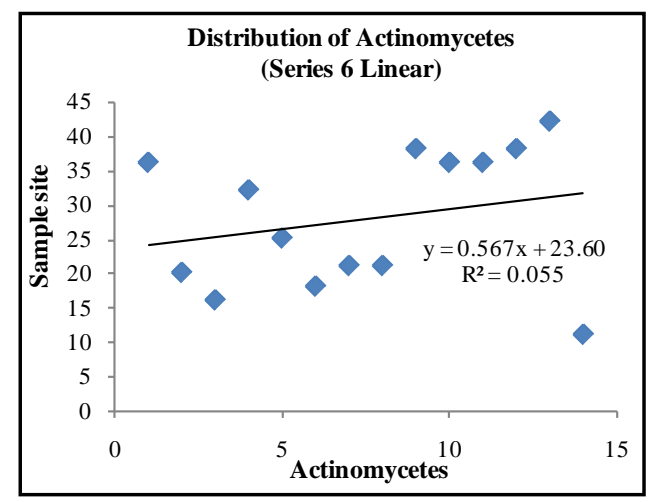

(g)

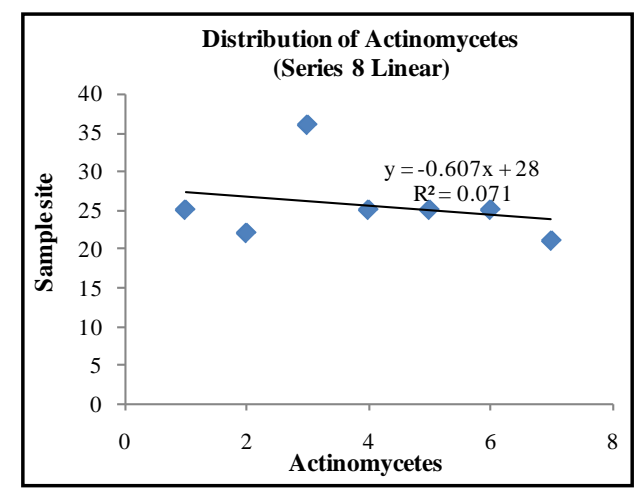

(i) 


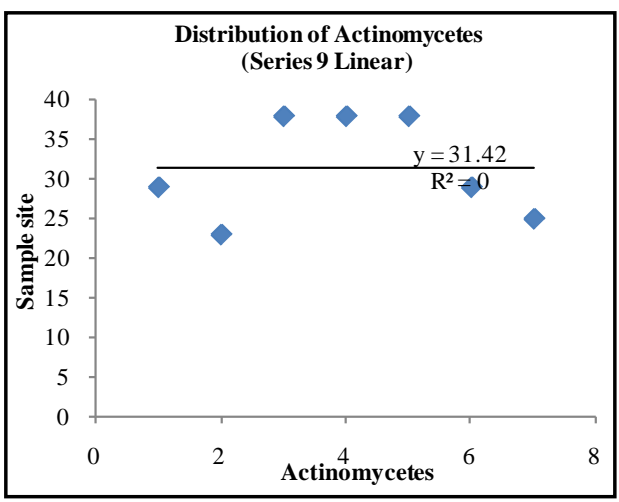

(j)

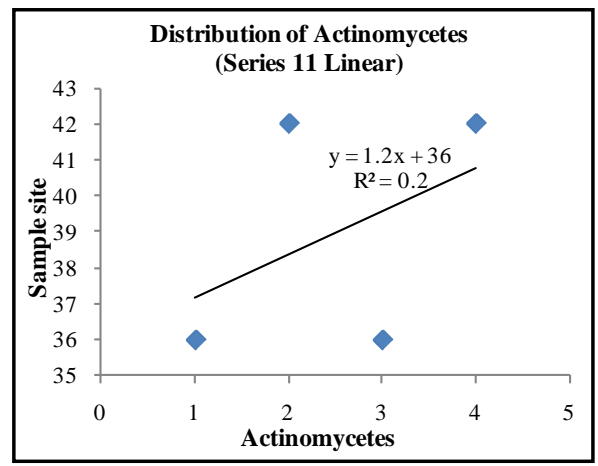

(l)

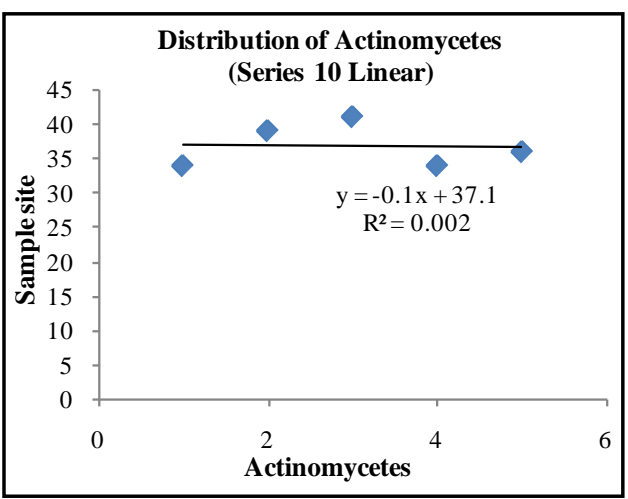

(k)

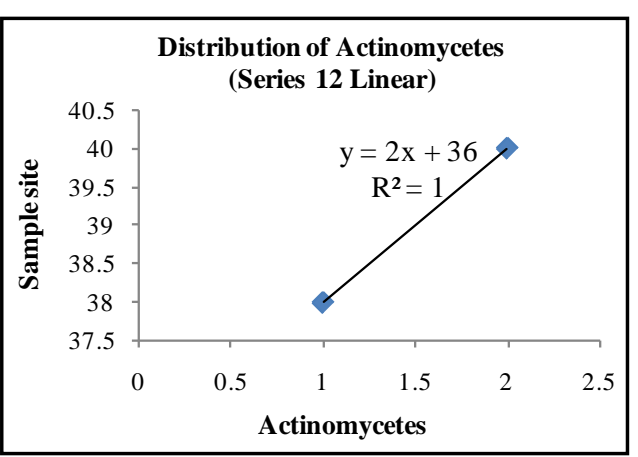

(m)

Figure 4. Represents the distribution of Actinomycetes isolated from different sampling sites. Distribution map shows 12 Series (Figures 4(b)-(m)). The trend line equation for each series are Series 1; $\mathrm{Y}=0.0502 \mathrm{x}+23.417, \mathrm{R} 2=0.0386$, Series $2 ; \mathrm{Y}=-0.3102 \mathrm{x}+31.576, \mathrm{R} 2=0.0809$, Series 3 ; $\mathrm{Y}=-0.4017 \mathrm{x}+32.981, \mathrm{R} 2=0.1246$, Series $4 ; \mathrm{Y}=-0.0371 \mathrm{x}+25.981, \mathrm{R} 2=0.0009$, Series $5 ; \mathrm{Y}=$ $0.0689 \mathrm{x}+24.299, \mathrm{R} 2=0.0019$, Series $6 ; \mathrm{Y}=0.567 \mathrm{x}+23.604, \mathrm{R} 2=0.0559$, Series $7 ; \mathrm{Y}=$ $-0.5515 \mathrm{x}+29.533, \mathrm{R} 2=0.0368$, Series $8 ; \mathrm{Y}=0.6071 \mathrm{x}+28, \mathrm{R} 2=0.0718$, Series $9 ; \mathrm{Y}=31.429$, $\mathrm{R} 2=0.0$, Series $10 ; \mathrm{Y}=-0.01 \mathrm{x}+37.1, \mathrm{R} 2=0.0026$, Series $11 ; \mathrm{Y}=1.2 \mathrm{x}+36, \mathrm{R} 2=0.2$ and Series $12 ; \mathrm{Y}=2 \mathrm{x}+36, \mathrm{R} 2=1$. All these series are showing linear relationship. These series also matches with the frequency class (frequency of occurrence) as shown in Table 1.

\section{Acknowledgements}

We are thankful to Prof. Anil K. Gupta for honorary supervision and support to carry out this research. Authors are also thankful to Sadbhav Foundation Mumbai, UNDP/GEF and Department of Science \& Technology (DST) New Delhi for partial funding of the project. We are deeply indebted for the help of Dr. P Yellore, SameersinghParmar, Dilip Koradia, Ramji Dabhi, Chiman Parmar, Jayendra Vaghela and Devshi Desai during the project period.

\section{References}

[1] Vining, L.C. (1992) Secondary Metabolism, Inventive Evolution and Biochemical Diversity-A Review. Gene, 115, 135-140. http://dx.doi.org/10.1016/0378-1119(92)90551-Y

[2] Edwards, C. (1993) Isolation, Properties and Potential Application of Thermophilicactinomycetes. Applied Biochemistry and Biotechnology, 42, 161-179.

[3] Demain, A.L. (1995) Why Do Microorganisms Produce Antimicrobials? In: Hunter, P.A., Darby, G.K. and Russell, N.J., Eds., Fifty Years of Antimicrobials: Prospective and Future Trends-Symposium 53, Society of General Microbiology, Cambridge University Press, Cambridge, 205-228.

[4] Jiang, Y., Chen, X., Lou, K. and Mao, P. (2013) Cultivable Soil Actinomycete Communities in Some Areas of Western China. Academy Journal of Biotechnology, 1, 1-13.

[5] Basilio, A., Gonzalez, I., Vicente, M.F., Gorrochategui, J., Cabello, A., Gonzalez, A. and Genilloud, O. (2003) Patterns 
of Antimicrobial Activities from Soil Actinomycetes Isolated under Different Conditions of pH and Salinity. Journal of Applied Microbiology, 95, 814-823. http://dx.doi.org/10.1046/j.1365-2672.2003.02049.x

[6] Moncheva, P., Tishkov, S., Dimitrova, N., Chipeva, V., Antonova-Nikolova, S. and Bogatzevska, N. (2000-2002) Characteristics of Soil Actinomycetes from Antarctica. Journal of Culture Collections, 3, 3-14.

[7] Miyadoh, S. (1997) Frontispiece. In: Miyadoh, S., Ed., Atlas of Actinomycetes, The Society for Actinomycetes Japan, Asakura Co., 1-5.

[8] Sahin, N. and Ugur, A. (2003) Investigation of the Antimicrobial Activity of Some Streptomyces Isolates. Turkish Journal of Biology, 27, 79-84.

[9] Kurtboke, D.I. (2005) Actinophages as Indicator of Actinomycete Taxa in Marine Environments. Anton van Leeuw, 87, 19-28. http://dx.doi.org/10.1007/s10482-004-6535-y

[10] Aghighi, S., Shahidi Bonjar, G.H., Rawashdeh, R., Batayneh, S. and Saadoun, I. (2004) First Report of Antifungal Spectra of Activity of Iranian Actinomycetes Strains against Alternariasolani, A. alternate, Fusariumsolani, Phytophthoramegasperma, Verticilliumdahliea and Saccharomyces cerevisiae. Asian Journal of Plant Sciences, 3, 463-471. http://dx.doi.org/10.3923/ajps.2004.463.471

[11] Vali, S., Suvathi, S.S., Ayusha, O.S., Nirmala, P., Vinoth, K.P. and Reena, A. (2012) Antimicrobial Potential of Actinomycetes Species Isolated from Marine Environment. Asian Pacific Journal of Tropical Biomedicine, 469-473.

[12] Nanjwade, B.K., Chandrashekhara, S., Shamarez, A.M., Goudanavar, P.K. and Manvi, F.V. (2010) Isolation and Morphological Characterization of Antibiotic Producing Actinomycetes. Tropical Journal of Pharmaceutical Research, 9, 231-236.

[13] Ouhdouch, Y., Barakate, M. and Finance, C. (2001) Actinomycetes of Moroccan Habitats: Isolation and Screening for Antifungal Activities. European Journal of Soil Biology, 37, 69-74. http://dx.doi.org/10.1016/S1164-5563(01)01069-X

[14] Tortorano, A.M., Cabrini, E. and Vivani, M.A. (1979) Sensibilite in Vitro des Levuresacinq Antibiotiques. Comparaison de deuxmethodes C.M.I. en gelose et methode des disques. Bulletin of Social Frame Mycal Medicine, 8, 69-74.

[15] Oskay, M., Tamer, A.U. and Azeri, C. (2004) Antibacterial Activity of Some Actinomycetes Isolated from Farming Soils of Turkey. African Journal of Biotechnology, 3, 441-446.

[16] Ceylan, O., Okmen, G. and Ugur, A. (2008) Isolation of Soil Streptomyces as Source Antibiotics Active against Antibiotic-Resistant Bacteria. European and Asian Journal of BioSciences, 2, 73-82.

[17] Gurung, T.D., Sherpa, C., Agrawal, V.P. and Lekhak, B. (2009) Isolation and Characterization of Antibacterial Actinomycetes from Soil Samples of Kalapatthar, Mount Everest Region. Nepal Journal of Science and Technology, 10, 173-182.

[18] Kuchari, M.G.A. (1999) The Isolation of Halotolerant Pigmented Actinomycetes from Jeddah Sea Shore, Saudi Arabia. Journal of King Abdulaziz University, 11, 5-12.

[19] Lam, K.S. (2006) Discovery of Novel Metabolites from Marine Actinomycetes. Current Opinion in Microbiology, 9, 245-251. http://dx.doi.org/10.1016/j.mib.2006.03.004

[20] Bredholt, H., Fjaervic, E., Johnsen, G. and Zotchev, S.B. (2008) Actinomycetes from Sediments in the Trondheim Fjord, Norway: Diversity and Biological Activity. Marine Drugs, 6, 12-24. http://dx.doi.org/10.3390/md6010012

[21] Suthindhiran, K. and Kannabiran, K. (2009) Cytotoxic and Antimicrobial Potential of Actinomycetes Species Saccharopolyspora salina VITSDK4 Isolated from the Bay of Bengal Coast of India. American Journal of Infectious Diseases, 5, 90-98. http://dx.doi.org/10.3844/ajidsp.2009.90.98

[22] Araujo, J.M., Silva, A.C. and Azevedo, J.L. (2000) Isolation of Endophytic Actinomycetes from Roots and Leaves of Maize (Zea mays L.). Brazilian Archives of Biology and Technology, 43, 447-451. http://dx.doi.org/10.1590/S1516-89132000000400016

[23] Inderiati, S. and Franco, C.M.M. (2008) Isolation and Identification of Endophytic Actinomycetes and Their Antifungal Activity. Jounal of Biotechnology Research in Tropical Region, 1, 1-6.

[24] Watanabe, Y., Shinzato, N. and Fukatsu T. (2003) Isolation of Actinomycetes from Termites’ Guts. Bioscience, Biotechnology and Biochemistry, 67, 1797-1801. http://dx.doi.org/10.1271/bbb.67.1797

[25] Skoko, N., Vujovic, J., Savic, M., Papic, N., Vasiljevic, B. and Ljubijankic, G. (2005) Construction of Saccharomyces cerevisiae Strain FAV20 Useful in Detection of Immunosuppressants Produced by Soil Actinomycetes. Journal of Microbiological Methods, 61, 137-140. http://dx.doi.org/10.1016/j.mimet.2004.11.007

[26] Busti, E., Monciardini, P., Cavaletti, L., Bamonte, R., Lazzarini, A., Sosio, M. and Donadio, S. (2006) AntibioticProducing Ability by Representatives of a Newly Discovered Lineage of Actinomycetes. Microbiology, 152, 675-683. http://dx.doi.org/10.1099/mic.0.28335-0

[27] Sessitsch, A., Weilharter, A., Gerzabek, M.H., Kirchmann, H. and Kandeler, E. (2001) Microbial Population Structures in Soil Particle Size Fractions of a Long-Term Fertilizer Field Experiment. Applied and Environmental Microbiology, 


\section{7, 4215-4224. http://dx.doi.org/10.1128/AEM.67.9.4215-4224.2001}

[28] Vijaykumar, R., Muthukumar, C., Thajuddin, N., Panneerselvam, A. and Saravanamuthu, R. (2007) Studies on the Diversity of Actinomycetes in the Palk Strait Region of Bay of Bangal, India. Actinomycetologica, 21, 59-65. http://dx.doi.org/10.3209/saj.SAJ210203

[29] Hong, K., Gao, A.H., Xie, Q.Y., Gao, H., Zhuang, L., Lin, H.P., Yu, H.P., Li, J., Yao, X.S., Goodfellow, M. and Ruan, J.S. (2009) Actinomycetes for Marine Drug Discovery Isolated from Mangrove Soils and Plants in China. Marine Drugs, 7, 24-44. http://dx.doi.org/10.3390/md7010024

[30] Pelaez, F. and Genilloud, O. (2003) Discovering New Drugs from Microbial Natural Products. In: Barredo, J.L., Ed., Microorganisms for Health Care, Foods and Enzyme Production, Research Signpost, Trivandrum, 1-22. 